\title{
Rotating Arm Internally Can Change the Arthroscopic Diagnosis of a Partial-thickness Tear of the Subscapularis
}

\author{
Hyungsuk Kim, Hyun Seok Song ${ }^{\nwarrow /}$, Seung Gu Kang, Sung Bin Han \\ Department of Orthopedic Surgery, Eunpyeong St. Mary's Hospital, College of Medicine, The Catholic University of Korea, Seoul, Korea
}

Background: The aims of this study were (1) to examine the footprint of the subscapularis tendon using the traditional posterior portal and $30^{\circ}$ arthroscope by simple internal rotation of the arm during surgery, and (2) to classify the pattern of a subscapularis partial-thickness tear.

Methods: This study analyzed a total of 231 patients with a partial-thickness subscapularis tear from 550 consecutive patients undergoing an arthroscopic operation who had a visualization of the subscapularis tendon footprint by internal rotation of the arm. First, the patients were classified into four categories according to the tear pattern: (1) stable lamination, (2) unstable lamination, (3) avulsion, and (4) laminated avulsion. Randomized arthroscopic videos were reviewed blindly by two independent orthopedic surgeons. The pattern of the tear of the subscapularis at the neutral position and after internal rotating the arm were assessed and compared with the treatment decision (level IV case series).

Results: Stable lamination, unstable lamination, avulsion, and laminated avulsion were observed in 9.1\% $(n=21), 20.8 \%(n=48), 41.1 \%$ $(n=95)$, and $29.0 \%(n=67)$ of cases, respectively. In 145 out of 231 cases $(62.8 \%)$, the decision was changed after inspecting the footprint after internal rotation of the arm, and the treatment method was changed in $116(50.2 \%)$ cases.

Conclusions: In a subscapularis tendon partial-thickness tear, inspecting the footprint of the subscapularis tendon is essential to diagnosing and deciding on the appropriate treatment. In addition, simply internal rotating the arm during surgery when using the traditional posterior portal and $30^{\circ}$ arthroscope can be a valuable method.

(Clin Shoulder Elbow 2019;22(3):135-138)

Key Words: Rotator cuff; Subscapularis; Diagnosis; Arthroscopy

\section{Introduction}

Rotator cuff tears are one of the main causes of shoulder pain and dysfunction. The subscapularis tendon is the largest and strongest tendon among the rotator cuff. Tears that involve the subscapularis tendon are less common compared to supraspinatus or infraspinatus tears because of their unreliable predictability on preoperative magnetic resonance imaging (MRI) scans." On the other hand, the advance of arthroscopic shoulder surgery has led to an increasing incidence and diagnosis of subscapularis tears. The incidence of subscapularis tears was reported to be
$2.1 \%$ by Deutsch et al., ${ }^{2)} 10.5 \%$ by Flury et al., $27.4 \%$ by Arai et al., ${ }^{4)}$ and $43 \%$ by Burkhart and Tehrany. ${ }^{5}$ Viewing the footprint of the subscapularis tendon using a $30^{\circ}$ arthroscope through a standard posterior portal is difficult. A diagnosis of subscapularis tears on preoperative MRI scans has been reported. ${ }^{6,7}$ Overall, these reports can explain the wide variation of incidences reported. $^{8,9)}$

The subscapularis tendon's footprint has a trapezoid shape, ${ }^{10)}$ but Arai et al. ${ }^{11)}$ reported a relatively thin and long insertion. Most partial-thickness tears of the subscapularis occur at the cephalad portion of the tendon and the most superior insertion

Received August 4, 2019. Revised August 11, 2019. Accepted August 11, 2019.

Correspondence to: Hyun Seok Song

Department of Orthopedic Surgery, Eunpyeong St. Mary's Hospital, College of Medicine, The Catholic University of Korea, 1021 Tongil-ro, Eunpyeong-gu, Seoul 03312, Korea

Tel: +82-2-2030-4628, Fax: +82-2-2030-4629, E-mail: hssongmd@yahoo.com, ORCID: https://orcid.org/0000-0002-7844-2293

IRB approval: The Catholic University of Korea, St. Paul's Hospital (No. PC14RISI0055).

Financial support: None. Conflict of interests: None. 
point of the tendon function as a buttress to support the superior glenohumeral ligament to stabilize the long head of the biceps tendon. The diagnosis of a partial-thickness tear of the subscapularis tendon is difficult in standard diagnostic shoulder arthroscopy because the mean height of the visible portion of the tendon is approximately $44 \%$ of the overall height and it is impossible to view the footprint on the lesser tuberosity of the humerus. ${ }^{12,13)}$

The current classifications of subscapularis tears are based on full thickness tears according to size and retraction introduced by Ide et al., ${ }^{14)}$ and partial-thickness tears by Arai et al. ${ }^{4)}$ classified by the tear pattern, such as longitudinal, transverse, and longitudinal plus transverse. On the other hand, there are no clinical reports or treatment guidelines using these classifications.

The difference in incidence reported on partial-thickness tears of the subscapularis tendon and the absence of established classification and treatment guidelines explains that with the standard arthroscope examination, diagnosis and treatment decision are difficult to make because of the limitation of the subscapularis tendon view, as mentioned above.

The aims of this study were (1) to analyze the efficacy of internal rotation of the arm during surgery to evaluate the footprint of the subscapularis tendon using the traditional posterior portal and $30^{\circ}$ arthroscope, (2) to classify the pattern of subscapularis partial-thickness tear, and (3) to determine if an examination of the footprint by internally rotating the arm affects the treatment decision.

\section{Methods}

\section{Inclusion}

Five-hundred fifty consecutive patients undergoing an arthroscopic operation, who had a visualization of the subscapularis tendon footprint by internal rotation of the arm were first analyzed retrospectively using recorded operation videos. The inclusion criterion was a partial-thickness tear of the subscapularis tendon. The exclusion criteria were a complete tear of the subscapularis tendon or normal. The operation was performed in the beach-chair position and the standard posterior portal was used with a $30^{\circ}$ arthroscope. First, the subscapularis tendon was
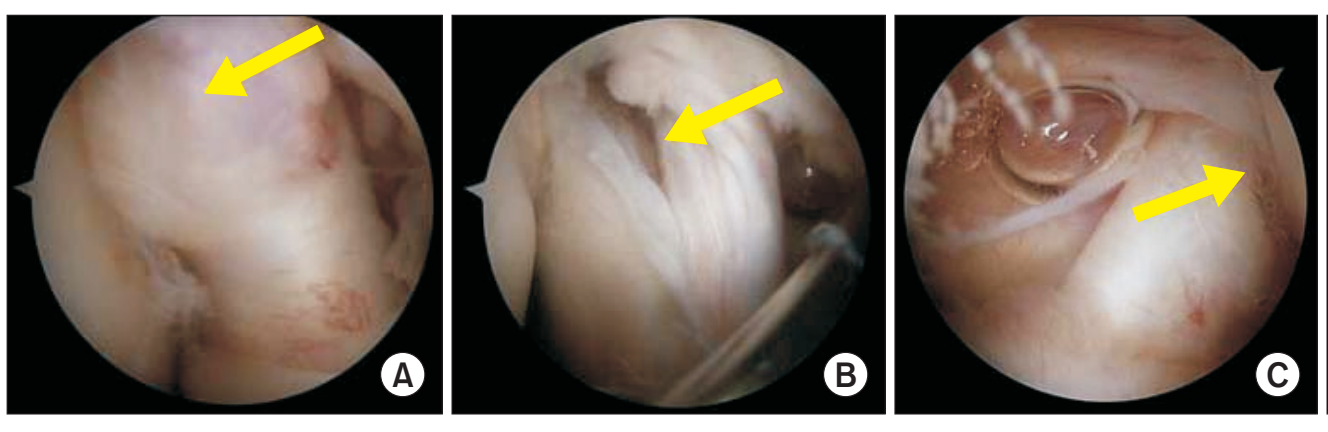

Fig. 1. Arthroscopic images showing stable lamination (A), unstable lamination (B), avulsion (C), and laminated avulsion (D) (arrows are indicating the tears).

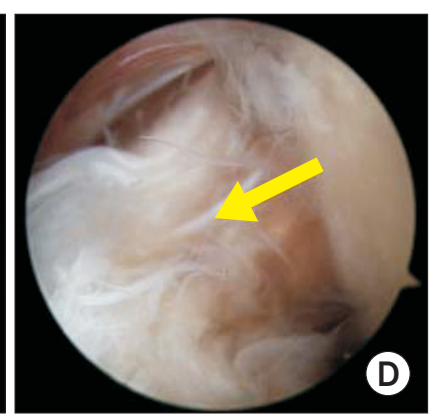

viewed with the arm in the neutral position and the footprint was then viewed after internal rotation of the arm. Two hundred and thirty one consecutive patients were confirmed to have a partial-thickness subscapularis tear and were analyzed.

The tear patterns were analyzed dynamically by internal rotation of the arm and classified into four types according to whether the sheath at the leading edge of the subscapularis tendon was torn and the footprint was exposed (Fig. 1).

First, when the tear was present at the tendon sheath, it was classified as lamination and then classified further as unstable lamination or stable lamination regarding whether buckling was present or not after internal rotation of the arm. A classification of avulsion was made when the tendon sheath was intact but a partial-thickness tear was found on the footprint after internally rotating the arm. When tear was found on both the tendon sheath and on the footprint, it was classified as laminated avulsion. When repairing the tendon, the author used a suture anchor when the footprint exposure was more than $50 \%$ in the avulsion type and a simple knot in the case of the unstable laminated type.

\section{Efficacy of Internal Rotation of the Arm in Diagnosis and Decision}

Each arthroscopic video for all cases was randomized using a computer number generator and given to two independent orthopedic surgeons, who were blinded to the patient's diagnosis, information, and surgical findings.

First, through the standard posterior portal and the arm in the neutral position, the tear pattern was analyzed using the classification mentioned above and they were asked to decide the treatment option. The treatment method was observation, deassessed again after internally rotating the arm and the surgeon decided on the treatment option.

\section{Statistical Analysis}

The concordance between the two surgeons in the neutral and internal rotation position was analyzed using a correlation test. By comparing the neutral position and internal rotated posibridement, or repair. On the same patient, the tear pattern was 
tion, the frequency of changing the diagnosis or treatment option was analyzed. Statistical analysis was performed using SAS software ver. 9.3 (SAS Institute, Cary, NC, USA).

The Institutional Review Board (IRB) approved the study protocol. The requirement for informed consent was waived due to the retrospective nature of the study.

\section{Results}

\section{Tear Patterns}

The incidence of stable lamination, unstable lamination, avulsion, and laminated avulsion was $9.1 \%(n=21), 20.8 \%(n=48)$, 41.1\% ( $n=95)$, and $29.0 \%(n=67)$, respectively. The avulsion type, which was torn only on the footprint, was most common. In addition, the stable lamination type, which was torn only on the tendon sheath, was the least common.

\section{Change of Diagnosis of the Tear patterns}

The decision was changed after inspecting the footprint by internally rotating the arm in 145 of the 231 cases (62.8\%).

\section{Change of Treatment Decision}

The author performed debridement on stable lamination, repair using a simple knot on unstable lamination, and repair using a suture anchor if the tear was more than $50 \%$ of the insertion site. In 116 out of 231 cases (50.2\%), the treatment method was changed from debridement to repair.

\section{Discussion}

The subscapularis tendon was first viewed using the standard posterior portal and a reevaluation of the partial-thickness tear pattern and treatment decision was made after viewing the footprint of the subscapularis tendon by internal rotating the arm. Compared to the neutral position, the diagnosis of the tear pattern was changed in $62.8 \%$ and the treatment decision was changed in $50 \%$.

Lamination and avulsion were classified because through the standard posterior portal view, a misdiagnosis can occur when the tendon sheath of the subscapularis tendon is normal and the footprint of the tendon is torn. Indeed, there were 95 cases (41.1\%) of the avulsion type, in which the tendon sheath is intact and the footprint is torn, which was the most common. Lamination, in which only the tendon sheath is torn, and laminated avulsion, in which both the tendon sheath and the footprint are torn, are classified in terms of clinical significance because the treatment changes from repair with a simple knot to repair using a suture anchor. ${ }^{15,16)}$ In this study, there were 67 cases (29.0\%) of laminated avulsion, which was the second most common. A tear of the subscapularis tendon on the footprint was observed in 162 cases $(70.1 \%)$ after internally rotating the arm. Hence, it is important to examine the footprint of the subscapularis tendon in the internal rotation position to make a correct decision. In addition, the lamination type was classified as stable lamination and unstable lamination because the treatment is debridement for the former and repair with a simple knot for the latter. Therefore, the classification introduced in this study is helpful for deciding the appropriate surgical treatment.

The currently introduced classification of a subscapularis tear includes classification by Pfirrmann et al. ${ }^{17)}$ using the MRI findings; Nérot et al. ${ }^{18)}$ and Walch et al. ${ }^{19)}$ proposed classifications for lesions that extend to the rotator interval in open findings, and Ide et al. ${ }^{14)}$ classified the full thickness tear of subscapularis tendon by open findings in a cadaveric study according to the size and the degree of retraction. Using the arthroscope, Bennett ${ }^{20)}$ classified a subscapularis tendon tear according to the thickness, length, retraction, and involvement of the near ligaments, and Arai et al. ${ }^{4}$ classified a partial-thickness tear of the subscapularis tendon by pattern viewing from the standard posterior portal. On the other hand, these classifications have limitations because they do not include the view of the footprint. Throughout the results of this study, the classification introduced by the author has clinical significance because viewing the footprint of the subscapularis tendon plays a major role in diagnosing and making a treatment decision.

The footprint of the subscapularis tendon can be observed using a $70^{\circ}$ arthroscope. ${ }^{21)}$ On the other hand, the use of an additional arthroscope can increase the cost and changing the arthroscopes during surgery may be troublesome and take more time.

This study had several limitations. The most obvious weakness was the retrospective collection of data. In addition, this study did not analyze the clinical results after deciding the treatment. Therefore, it is impossible to know whether the treatment decided by the classification introduced was appropriate. Because the patients included in this study did not have an isolated partial-thickness subscapularis tear and some were treated for other concomitant shoulder lesions, these factors may affect the clinical results. Therefore, the limitations are unavoidable when analyzing clinical results.

Despite these limitations, the strengths of this study are the large number of data included and the first classification introduced for partial-thickness subscapularis tendon tears that include the footprint. In addition, the footprint of the subscapularis tendon was viewed by a $30^{\circ}$ arthroscope by simple internal rotation of the arm during surgery, thereby avoiding the trouble of changing to a $70^{\circ}$ arthroscope.

\section{Conclusion}

In subscapularis tendon partial-thickness tears, it is clinically important to evaluate the footprint of the subscapularis tendon 
when using a traditional posterior portal. In 145 out of 231 cases $(62.8 \%)$, the decision was changed after inspecting the footprint after internal rotation of the arm. In 116 cases (50.2\%), the treatment method was changed from debridement to repair. Overall, simple internal rotation of the arm during surgery when using the traditional posterior portal and $30^{\circ}$ arthroscope can be a valuable method.

\section{References}

1. Tung GA, Yoo DC, Levine SM, Brody JM, Green A. Subscapularis tendon tear: primary and associated signs on MRI. J Comput Assist Tomogr. 2001;25(3):417-24. doi: 10.1097/ 00004728-200105000-00015.

2. Deutsch A, Altchek DW, Veltri DM, Potter HG, Warren RF. Traumatic tears of the subscapularis tendon. Clinical diagnosis, magnetic resonance imaging findings, and operative treatment. Am J Sports Med. 1997;25(1):13-22. doi: 10.1177/ 036354659702500104.

3. Flury MP, John M, Goldhahn J, Schwyzer HK, Simmen BR. Rupture of the subscapularis tendon (isolated or in combination with supraspinatus tear): when is a repair indicated? J Shoulder Elbow Surg. 2006;15(6):659-64. doi: 10.1016/ j.jse.2005.07.013.

4. Arai R, Sugaya H, Mochizuki T, Nimura A, Moriishi J, Akita K. Subscapularis tendon tear: an anatomic and clinical investigation. Arthroscopy. 2008;24(9):997-1004. doi: 10.1016/ j.arthro.2008.04.076.

5. Burkhart SS, Tehrany AM. Arthroscopic subscapularis tendon repair: technique and preliminary results. Arthroscopy. 2002;18(5):454-63. doi: 10.1053/jars.2002.30648.

6. Cash CJ, MacDonald KJ, Dixon AK, Bearcroft PW, Constant CR. Variations in the MRI appearance of the insertion of the tendon of subscapularis. Clin Anat. 2009;22(4):489-94. doi: 10.1002/ca.20789.

7. Adams CR, Schoolfield JD, Burkhart SS. Accuracy of preoperative magnetic resonance imaging in predicting a subscapularis tendon tear based on arthroscopy. Arthroscopy. 2010;26(11):1427-33. doi: 10.1016/j.arthro.2010.02.028.

8. Gerber C, Krushell RJ. Isolated rupture of the tendon of the subscapularis muscle. Clinical features in 16 cases. J Bone Joint Surg Br. 1991;73(3):389-94.

9. Walch G, Nove-Josserand L, Levigne C, Renaud E. Tears of the supraspinatus tendon associated with "hidden" lesions of the rotator interval. J Shoulder Elbow Surg. 1994;3(6):353-60. doi: 10.1016/S1058-2746(09)80020-7.
10. Curtis AS, Burbank KM, Tierney JJ, Scheller AD, Curran $A R$. The insertional footprint of the rotator cuff: an anatomic study. Arthroscopy. 2006;22(6):609.e1. doi: 10.1016/ j.arthro.2006.04.001.

11. Arai R, Mochizuki T, Yamaguchi K, et al. Functional anatomy of the superior glenohumeral and coracohumeral ligaments and the subscapularis tendon in view of stabilization of the long head of the biceps tendon. J Shoulder Elbow Surg. 2010;19(1):58-64. doi: 10.1016/j.jse.2009.04.001.

12. Wright JM, Heavrin B, Hawkins RJ, Noonan T. Arthroscopic visualization of the subscapularis tendon. Arthroscopy. 2001;17(7):677-84. doi: 10.1053/jars.2001.25274.

13. Koo SS, Burkhart SS. Subscapularis tendon tears: identifying mid to distal footprint disruptions. Arthroscopy. 2010;26(8):1130-4. doi: 10.1016/j.arthro.2010.06.017.

14. Ide J, Tokiyoshi A, Hirose J, Mizuta H. An anatomic study of the subscapularis insertion to the humerus: the subscapularis footprint. Arthroscopy. 2008;24(7):749-53. doi: 10.1016/ j.arthro.2008.02.009.

15. Adams CR, Schoolfield JD, Burkhart SS. The results of arthroscopic subscapularis tendon repairs. Arthroscopy. 2008;24(12):1381-9. doi: 10.1016/j.arthro.2008.08.004.

16. Kim SH, Oh I, Park JS, Shin SK, Jeong WK. Intra-articular repair of an isolated partial articular-surface tear of the subscapularis tendon. Am J Sports Med. 2005;33(12):1825-30. doi: 10.1177/0363546505278259.

17. Pfirrmann CW, Zanetti M, Weishaupt D, Gerber C, Hodler J. Subscapularis tendon tears: detection and grading at MR arthrography. Radiology. 1999;213(3):709-14. doi: 10.1148/ radiology.213.3.r99dc03709.

18. Nérot C, Jully JL, Gérard Y. [Rotator cuff ruptures with predominant involvement of the subscapular tendon]. Chirurgie. 1993-1994;119:404-10. French.

19. Walch G, Boileau P, Noël E, Liotard JP, Dejour H. [Surgical treatment of painful shoulders caused by lesions of the rotator cuff and biceps, treatment as a function of lesions. Reflections on the Neer's concept]. Rev Rhum Mal Osteoartic. 1991;58(4):247-57. French.

20. Bennett WF. Subscapularis, medial, and lateral head coracohumeral ligament insertion anatomy. Arthroscopic appearance and incidence of "hidden" rotator interval lesions. Arthroscopy. 2001;17(2):173-80. doi: 10.1053/jars.2001.21239.

21. Burkhart SS, Brady PC. Arthroscopic subscapularis repair: surgical tips and pearls A to Z. Arthroscopy. 2006;22(9):1014-27. doi: 10.1016/j.arthro.2006.07.020. 